\title{
NCCN Evidence Blocks
}

\author{
Presented by Robert W. Carlson, MD, and Eric Jonasch, MD
}

\begin{abstract}
NCCN has developed a series of Evidence Blocks: graphics that provide ratings for each recommended treatment regimen in terms of efficacy, toxicity, quality and consistency of the supporting data, and affordability. The NCCN Evidence Blocks are currently available in 10 tumor types within the NCCN Clinical Practice Guidelines in Oncology (NCCN Guidelines). At a glance, patients and providers can understand how a given treatment was assessed by the NCCN Guidelines Panel and get a sense of how a given treatment may match individual needs and preferences. Robert W. Carlson, MD, CEO of NCCN, described the reasoning behind this new feature and how the tool is used, and Eric Jonasch, MD, Professor of Genitourinary Medical Oncology at The University of Texas MD Anderson Cancer Center, and Vice Chair of the NCCN Kidney Cancer Panel, described its applicability in the management of metastatic renal cell carcinoma.
\end{abstract}

J Natl Compr Canc Netw 2016;14(5.5):616-619

\section{NCCN Evidence Blocks to Guide Decision-Making}

NCCN recently introduced NCCN Evidence Blocks into a number of the NCCN Clinical Practice Guidelines in Oncology (NCCN Guidelines) to assist clinicians and patients as they grapple with treatment choices among an ever-growing number of options and as the cost, of oncology treatment becomes of increasing concern. The NCCN Evidence Blocks are graphic elements that weight several relevant factors in choice of cancer treatment, including cost, and show these weights against each other. According to Robert W. Carlson, MD, CEO of NCCN, clinicians, patients, and payers alike have expressed the desire to better understand the relative efficacy, toxicity, and costs of treatment.

Presented by Robert W. Carlson, MD, NCCN, Fort Washington, Pennsylvania, and Eric Jonasch, MD, The University of Texas MD Anderson Cancer Center, Houston, Texas.

Robert W. Carlson, MD, has disclosed that he is the Chief Executive Officer of NCCN, but otherwise has no financial interests, arrangements, affiliations, or commercial interests with the manufacturers of any products discussed in this article or their competitors. Eric Jonasch, MD, has disclosed that he has received consulting fees from Bristol-Myers Squibb Company, Exelixis Inc., Novartis Pharmaceuticals Corporation, Pfizer Inc., and Roche Laboratories, Inc.; honoraria from Bristol-Myers Squibb Company, Novartis Pharmaceuticals Corporation, Pfizer Inc., and Roche Laboratories, Inc.; and grant/research support from Exelixis Inc., Novartis Pharmaceuticals Corporation, Onyx Pharmaceuticals, Inc., and Pfizer Inc.

Correspondence: Robert W. Carlson, MD, NCCN, 275 Commerce Drive, Suite 300, Fort Washington, PA 19034. E-mail: carlson@nccn.org Eric Jonasch, MD, The University of Texas MD Anderson Cancer Center, 1515 Holcombe Boulevard, Unit 1374, Houston, TX 77030.

E-mail: ejonasch@mdanderson.org
But cost is just part of determining the "value" of a given treatment, a concept for which awareness is growing and which the NCCN Evidence Blocks seek to address, Dr. Carlson said in describing the program at the NCCN 21st Annual Conference. He was joined by Eric Jonasch, MD, Professor of Genitourinary Medical Oncology, The University of Texas MD Anderson Cancer Center, and Vice Chair of the NCCN Kidney Cancer Panel, who described how this tool can be used in managing metastatic renal cell carcinoma (RCC).

\section{Evidence Encapsulated in a Simple Graphic}

The NCCN Evidence Blocks provide a number of measures by which to evaluate a treatment: efficacy, safety, quality and consistency of evidence supporting the recommended therapy, and affordability. All of this information is presented in the form of a graphic for ease of use and understanding (Figure 1).

Although most of these factors have been part of decision-making for some time, cost is a more recent consideration. The expense of a given treatment is not integral to any recommendation made by NCCN; however, cost is an increasingly important component of care that can no longer be ignored, Dr. Carlson said. "Affordability," as the measure is called, refers to the overall cost of an intervention, including the cost for the drug, infusions, supportive care, toxicity monitoring and management, and the probability of care being delivered in the hospital. 


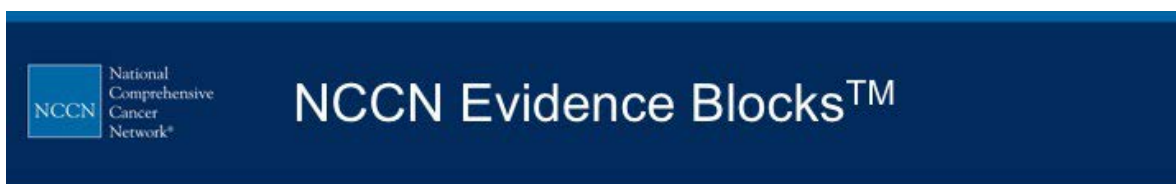

- Use consistent methodology and display to inform decision-making

- Measures

- Efficacy

- Safety

- Quality of Evidence

- Consistency of Evidence

- Affordability

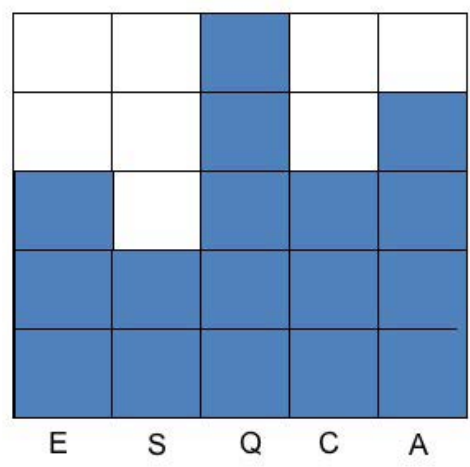

- More shading is better

Figure 1. NCCN Evidence Blocks.

"Cost is not considered in the NCCN Guidelines, but should be available to the patient in making treatment decisions," according to Dr. Carlson.

The scoring system is simple: shading of only 1 block indicates "least favorable," while 5 shaded blocks indicate "most favorable." The more shading in the block, the better the regimen in all components. The goal of the simple graphic is to allow clinicians and patients alike to quickly appreciate the relative merits of the treatment options. A quicktake on each regimen-facilitated by simple, intuitive graphics-helps patients and providers key-in on the factors that are most important them.

"Value has many definitions and varies greatly from patient to patient," Dr. Carlson said. "We are providing information that allows the patient to create the value formula, which is important in shared decision-making."

He said the NCCN Evidence Blocks are not prescriptive, but they are a starting point for a productive conversation between patient and physician.

\section{Available for 10 Tumor Sites}

Each evidence block is compiled from surveys completed by panel members, using the 5 measures for each treatment recommendation. Responses are collated, and an average score for each regimen and each measure is generated. The results are translated into the graphic, and the relevant NCCN Evidence Blocks are placed in the NCCN Guidelines algorithms.

Currently, there are versions of the NCCN Guidelines with NCCN Evidence Blocks for breast cancer, chronic myelogenous leukemia, colon cancer, kidney cancer, melanoma, multiple myeloma, diffuse large B-cell lymphoma, non-small cell lung cancer, prostate cancer, and rectal cancer.

Dr. Carlson noted that clinicians have expressed interest in getting more rationale for the NCCN Guideline recommendations. Patients have requested information to enhance decision-making. And, in response to the rising cost of cancer care, many groups have wanted more information on relative affordability.

Dr. Carlson acknowledged that the NCCN Evidence Blocks are not the only value-oriented tool for decision-making. ASCO's Value Framework calculates the added benefit from a new cancer drug compared with standard of care ("net health benefit"), and Memorial Sloan Kettering Cancer Center's "drug abacus" estimates the value-based cost of 51 oncology agents based on anticipated outcomes and economic 
Carlson and Jonasch

variables. The Institute for Clinical and Economic Review also has a "value-based price benchmark."

\section{Evidence Blocks Applied to RCC}

"Evidence Blocks are beginning to offer a succinct interpretation of the data, which can begin to generate a dialog between patients and their treatment teams," said Dr. Jonasch, who reviewed the treatment options for metastatic RCC in the NCCN Guidelines and showed how they are reflected in the NCCN Evidence Blocks.

Although no agents are available for the adjuvant treatment of resected primary RCC, multiple agents are available for metastatic disease, including the antiangiogenic agents sunitinib, pazopanib, bevacizumab/interferon, sorafenib, axitinib, and recently approved cabozantinib. The mTOR inhibitors include temsirolimus and everolimus, and immunomodulatory agents include the older drug interleukin-2 (IL-2) and now nivolumab.

A number of questions remain regarding the use of these drugs, including the best frontline treatment, ideal sequence of agents, and current role of mTOR inhibition.

"Here's how we contextualize the frontline treatments using the Evidence Blocks," Dr. Jonasch said
(Figure 2). "We see there is definitely some diversity in how these boxes have been filled out. The efficacy ranges between 2 boxes [temsirolimus] and 5 boxes [sunitinib, pazopanib, high-dose IL-2]. Similarly, the consistency of evidence and quality of evidence ranges from "extremely high to fairly good," according to the shading, he added.

\section{How Trial Data Influenced Ratings}

Dr. Jonasch focused on sunitinib, temsirolimus, and pazopanib to show how the data influenced these designations. Sunitinib was approved in the frontline setting by showing unprecedented progressionfree survival (11 months) compared with interferon (5 months) (hazard ratio [HR], 0.42; $\mathrm{P}<.001) .{ }^{1} \mathrm{Al}$ though overall survival was of borderline significance, it has since been established that patients receiving antiangiogenic agents do experience a survival benefit, he said.

Similarly, pazopanib resulted in a significant improvement in progression-free survival, versus placebo, in both untreated patients (9.2 vs 4.2 months; $P<.0000001)$ and pretreated patients (11.1 vs 2.8; $P<.0000001)$ in a phase III trial. ${ }^{2}$ The noninferiority COMPARZ trial compared pazopanib with sunitinib and showed comparable outcomes, "with virtually

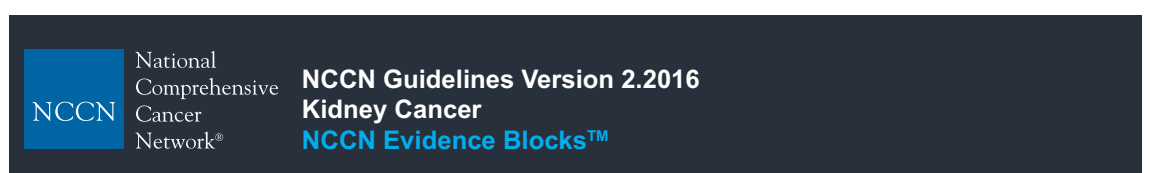

FIRST-LINE THERAPY
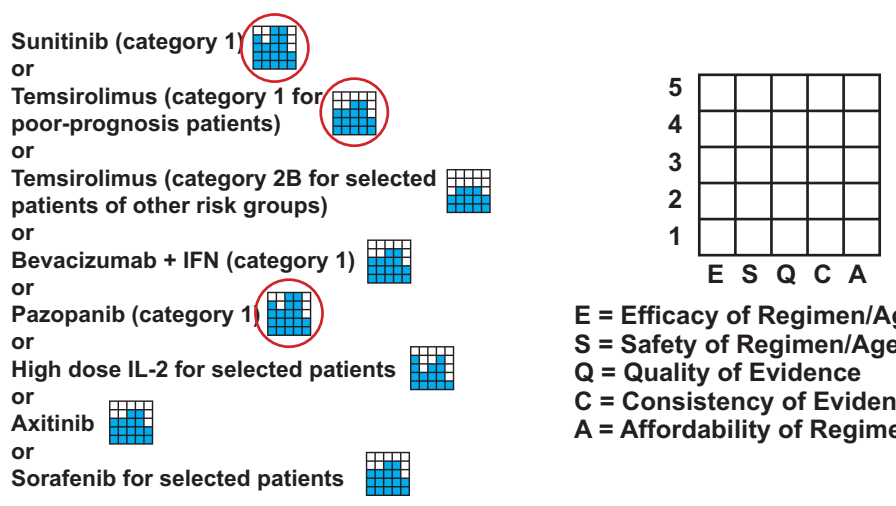

$E=$ Efficacy of Regimen/Agent

$\mathrm{S}=$ Safety of Regimen/Agent

$Q=$ Quality of Evidence

$\mathrm{C}=$ Consistency of Evidence

A = Affordability of Regimen/Agent

Sorafenib for selected patients

KID-3 
overlapping curves" for progression-free and overall survival. ${ }^{3}$ Median overall survival was approximately 29 months for each.

"The Evidence Blocks are congruent with the strength of evidence with these agents, and we see quite good efficacy," Dr. Jonasch said. For adverse events, pazopanib had a slight advantage, though the differences were not substantial and the panel scored safety as similar between the drugs.

"Because of the rapidity with which agents have become available for metastatic RCC, it's been difficult to extract an overall survival signal from the individual trials. Overall, however, we see survival increasing since the interferon era," he added.

A survival difference was shown, however-albeit modest - with one of the earlier agents, temsirolimus, which was approved in 2007. FDA approval was based on a randomized phase III trial in which median survival was 10.9 months versus 7.1 months with interferon. ${ }^{4}$

"The difference between this and other studies in metastatic RCC was that it included patients with predominantly intermediate- or poor-risk features, and it still achieved an overall survival benefit," he said. "The outcome measures were rated as somewhat more modest for this agent, but it still demonstrated reasonable efficacy."

In the second line, options include the older drugs everolimus, axitinib, cabozantinib, and nivolumab, all with category 1 recommendations. Also acceptable are sorafenib, sunitinib, pazopanib, temsirolimus, and bevacizumab. Of all these, the strongest efficacy-demonstrated by shading of 4 of the 5 blocks - is with nivolumab. The others are shown to be "modestly effective." In terms of tolerability, axitinib, everolimus, and cabozantinib are equivalent and nivolumab is scored slightly higher. The 4 agents are similar in terms of affordability, Dr. Jonasch indicated.

The key question to be explored next is the optimal sequence of these agents; that is, should an antiangiogenic agent be given first and, if so, which agent comes next? "This is the big question we are all grappling with," Dr. Jonasch said. "Also, we need to get our heads around what defines the quality of a response and relative toxicity? And how do we measure affordability? It's critical to begin talking about these issues."

These discussions, he added, will lead to ongoing refinement of the NCCN Evidence Blocks.

\section{References}

1. Motzer RJ, Hutson TE, Tomczak P, et al. Sunitinib versus interferon alfa in metastatic renal-cell carcinoma. N Engl J Med 2007;356:115-124.

2. Sternberg CN, Davis ID, Mardiak J, et al. Pazopanib in locally advanced or metastatic renal cell carcinoma: results of a randomized phase III trial. J Clin Oncol 2010;28:1061-1068.

3. Motzer RJ, Hutson TE, McCann L, et al. Overall survival in renal-cell carcinoma with pazopanib versus sunitinib. N Engl J Med 2014;370:17691770 .

4. Hudes G, Carducci M, Tomczak P, et al. Temsirolimus, interferon alfa, or both for advanced renal-cell carcinoma. N Engl J Med 2007;356:2271-2281. 